\title{
Non-destructive, safe removal of conductive metal coatings from fossils: a new solution
}

\author{
David Jones, Jennifer Hartley, Gero Frisch, Mark Purnell, and Laurent Darras
}

\begin{abstract}
Scanning electron microscopy, and some other imaging techniques, commonly require that specimens to be imaged are coated with a conductive metal, such as gold, gold-palladium, platinum or silver. However, the application of metal coatings changes the appearance of specimens and can obscure important features, and thus may be undesirable, or even prohibited by institutions or curators. We describe a harmless, straightforward and inexpensive technique for removing gold. The method involves immersing samples in ionic liquids and rinsing in water. No further handling is needed, no poisonous compounds are utilised in the process, and the liquids may be tailored to remove other metal coatings without affecting the adhesive used to attach the specimen to the substrate.
\end{abstract}

David Jones. School of Earth Sciences, University of Bristol, Wills Memorial Building,

Queen's Road, Bristol, BS8 1RJ, United Kingdom. David.Jones@bristol.ac.uk

Jennifer Hartley. Department of Chemistry, University of Leicester, Leicester, LE1 7RH, United Kingdom.

jmh50@leicester.ac.uk

Gero Frisch. Department of Chemistry, University of Leicester, Leicester, LE1 7RH, United Kingdom.

gf46@leicester.ac.uk

Mark Purnell (correspondence author). Department of Geology, University of Leicester, Leicester, LE1

7RH, United Kingdom. map2@le.ac.uk

Laurent Darras. Department of Geology, University of Leicester, Leicester, LE1 7RH, United Kingdom.

Id101@le.ac.uk

KEY WORDS: microfossil; metal coating; SEM; ionic liquids

\section{INTRODUCTION}

An increasing diversity of techniques for both producing 2D images and acquiring 3D data is currently applied to biological, geological and particularly palaeontological specimens. Many of these methods require that specimens are coated with a thin layer of metal: in particular, for scanning electron microscopy, to prevent charging and ensure that the surface conducts evenly (Miller et al., 2004; Leslie and Mitchell, 2007); but also in focus variation optical microscopy, to provide a reflective surface for translucent or transparent specimens (Purnell and Jones, in press; Purnell et al., 2012). 
However, coating can radically alter the appearance of specimens because it alters surface colour and renders translucent surfaces opaque. It can thus obscure significant features of a specimen. For example, well-preserved conodont elements are translucent and have internal structures, which can have anatomical and taxonomic importance, but these structures are hidden by coating. Furthermore, metal coatings are frequently problematic to remove: although the cyanide-based method reported by Leslie and Mitchell (2007) is an effective technique for removing gold and gold-palladium, some institutions express safety concerns at using the highly poisonous cyanide compounds involved (Mills, 1988; authors' personal experience). Consequently, institutions or curators are often reluctant to allow coating of specimens in their care. This is particularly true when they are rare or valuable. Thus, it is desirable to employ a safe approach to remove the coating without damaging the specimen.

Methods utilising less toxic substances have been outlined in the literature (Hansen, 1968; Crissman and McCann, 1979; Mills, 1988, 1989), as well as physical approaches (Golden, 1989; Miller et al., 2004), but we report here on a new technique with a number of benefits. First, it is flexible, because the liquids used can be tailored to remove a coating of any metal without affecting the specimen. Second, it does not affect the adhesives used to affix specimens to the substrate (for example SEM stubs), unless they are water soluble. This minimises the amount of specimen handling required, as complete mounted stubs, rather than individual specimens, can be treated. Third, it is inexpensive and straightforward, comprising just a few steps using a simple apparatus, and can be conducted on a laboratory bench top without a fume cupboard. Fourth, it is relatively quick, removing coating within $12 \mathrm{hr}$. We believe these advantages make this approach an attractive alternative for the removal of metal coatings and hope that it will increase the likelihood that institutions and curators will allow loaned specimens to be coated.

\section{Gold Oxidation}

Gold is a very inert metal. The chemical removal of a gold coating therefore requires an extremely strong oxidising agent, which could potentially damage the sample once the gold layer has been dissolved. Alternatively, the gold ions that are produced by the oxidation reaction can be stabilised with appropriate ligands (an ion/molecule bonding to a metal ion to produce a complex). Vari- ous cyanide-based methods employ this principle by stabilising gold ions in the form of cyano-complexes. Consequently, gold has a much lower redox potential in the presence of cyanide, i.e., oxidation becomes easier and moderate oxidising agents are sufficient.

In this work we present a method that employs iodine as a moderate oxidising agent and uses a benign 'deep eutectic solvent,' Ethaline, for gold ion stabilisation.

\section{Advantages of Ionic Liquids in Metallurgy}

lonic liquids are salts with a melting point below $100^{\circ} \mathrm{C}$. Deep eutectic solvents like Ethaline are a new class of ionic liquids. They have been designed as benign and cost effective matrices for metallurgical processes (Abbott et al., 2011a and citations therein). The ability of Ethaline to facilitate gold oxidation with iodine is based on two of its unusual properties: a high chloride concentration (ca. $5 \mathrm{~mol} \mathrm{dm}^{-3}$ ) and good solubility of iodine.

The exceptionally high concentration of chloride ions in the absence of other possibly complexing species generally promotes the formation of chloro-complexes when metal ions are dissolved in Ethaline. Late transition metals like palladium, platinum, silver and gold in particular form stable chloro-complexes and are therefore much affected by the chloride concentration in the solvent. Similar to the complexation with cyanide, their ionic form is stabilised by chloride in Ethaline and a moderate oxidising agent like iodine is sufficient to dissolve these otherwise inert metals. For other metals a similar effect can be achieved by judicious choice of the anionic component in the ionic liquid (see Abbott et al., 2011b for examples).

lodine is a benign and cost effective oxidising agent but has an insufficient solubility of only 0.29 $\mathrm{g} / \mathrm{kg}$ in water. Ethaline on the other hand can dissolve more than $200 \mathrm{~g}$ of iodine per $\mathrm{kg}$ of solvent and is therefore a good matrix for iodine-based oxidation. Classic organic solvents have similar properties with respect to iodine but, due to their nonpolar nature, do not stabilise metal ions.

\section{MATERIALS AND METHOD}

We selected specimens of different compositions to test the safety and efficacy of the technique in removing the substance most commonly used for coating: gold. We applied this method to several calcareous and phosphatic fossils and microfossils. The calcareous specimens were extant planktonic foraminifera, Globorotalia menardii, from the Caribbean Sea (2900 m depth). Phosphatic speci- 
mens included a range of vertebrate remains, including conodont elements (several morphologies, Silurian and Carboniferous), fragments of dermal armour from heterostracans and osteichthyans (Devonian), and scales, fin rays (lepidotrichia), and phosphatized muscle fibres from a lungfish (Onychodus, Devonian). These specimens provide a range of different tissue types with different susceptibilities to surface damage by chemical attack. Specimens were mounted on $12.7 \mathrm{~mm}$ diameter aluminium scanning electron microscope (SEM) stubs. In order to test the effect of solvents on common mounting adhesives, Agar Scientific double-sided carbon adhesive tabs, gum tragacanth and diluted PVA glue were used on different stubs. Specimens are curated in the Department of Geology collections at the University of Leicester (accession numbers LEIUG 123045-7).

The aim of our testing was to determine whether the solvents effectively removed a gold coating without affecting fossils of calcareous and phosphatic composition, and commonly used mounting adhesives. Many more tests with different combinations of variables could be conducted (e.g., fossils of silica or mixed compositions; conductive coatings of gold-palladium or silver; adhesives of different kinds, such as paraloid, double sides tape, nail varnish). Obviously, our analysis does not preclude further testing, but our results give us no reason to doubt that our technique would be equally effective and safe on a range of specimen compositions, coatings and adhesives (see Results and Discussion). Mounted stubs were sputter coated with approximately $30 \mathrm{~nm}$ thickness of gold (Emitech K500X and Bio Rad Microscience Division SC650). The appearance of whole specimens was recorded, using optical photomicrography, before coating and after removal using optical microscopy on either a Leica Microsystems DFC425C camera mounted on a Leica M205C microscope, or a Qimaging camera mounted on a Leica M8 microscope. Smaller areas of the specimens were also imaged at high magnification using scanning electron micrography (SE mode), to ensure there was no fine-scale damage.

Ethaline was prepared from a 1:2 (by moles) mixture of choline chloride (Sigma-Aldrich, reagent grade, $\geq 98 \%$ ) and ethylene glycol (Sigma-Aldrich, ReagentPlus grade, $\geq 99 \%$ ), which was heated and stirred at ca. $60^{\circ} \mathrm{C}$ until a clear homogenous liquid was obtained. This typically takes 10 to 15 minutes for a batch of $100 \mathrm{~g}$ but Ethaline can be made in any required amount. It is hygroscopic but can be stored for years in a closed glass or plastic (not metal) container, preferably in a dark place. At temperatures below $20^{\circ} \mathrm{C}$ choline chloride can precipitate from the liquid. In this case the mixture has to be heated and stirred again before use. lodine (Fisher, reagent grade, $\geq 99.5 \%$ ) was dissolved at concentrations of $0.02,0.05$ and $0.1 \mathrm{~mol} \mathrm{dm}^{-3}$ in this liquid under stirring at ca. $60^{\circ} \mathrm{C}$. The iodine solution is stable for a week and should be stored in a glass container (not plastic or metal). The preparation of the solvent and dissolution of iodine can also be achieved at room temperature but takes considerably longer. The iodine solution can be used several times, provided sufficient unreacted iodine is present, indicated by the solution remaining dark brown, but since the solution is both cheap and benign to dispose of, we used a fresh solution for every sample.

The gold-coated samples, i.e., specimens mounted on SEM stubs, were carefully placed using tweezers into a glass sample tube that contained ca. $20 \mathrm{~cm}^{3}$ of the above solution and left at ca. $60^{\circ} \mathrm{C}$ for times ranging from 30 minutes to 12 hours. Under the given conditions iodine was always in large excess. The volume of the solution and size of the sample tube depended on the size of the sample rather than the amount of gold to be removed. Subsequently, the samples were rinsed by placing them carefully in a saturated aqueous solution of potassium iodide (Fisher Scientific, reagent grade, $\geq 99 \%$ ), followed by rinsing in deionised water. Potassium iodide was used to increase the solubility of iodine in water, through the formation of $\mathrm{I}_{3}{ }^{-}$, and thus avoid precipitation of iodine on the samples. Rinsing the specimens was achieved by dipping the SEM stub into separate containers of each liquid, using tweezers, which is the most effective method. Careful handling in this manner is unlikely to result in the loss of specimens attached by non-water soluble adhesive, but alternatively a wash bottle or pipette can be used. A recipe for producing a standard quantity of the iodine solution is provided in the Appendix.

\section{RESULTS AND DISCUSSION}

Using the method described above, we have successfully removed gold coatings from phosphatic and calcareous microfossils, mounted on SEM stubs with carbon adhesive tabs, gum tragacanth or diluted PVA glue. Figures 1 and 2 show optical and SEM images of test specimens before coating and after gold removal using an iodine concentration of $0.02 \mathrm{~mol} \mathrm{dm}^{-3}$. Coatings were completely removed, and for durations of immersion up 


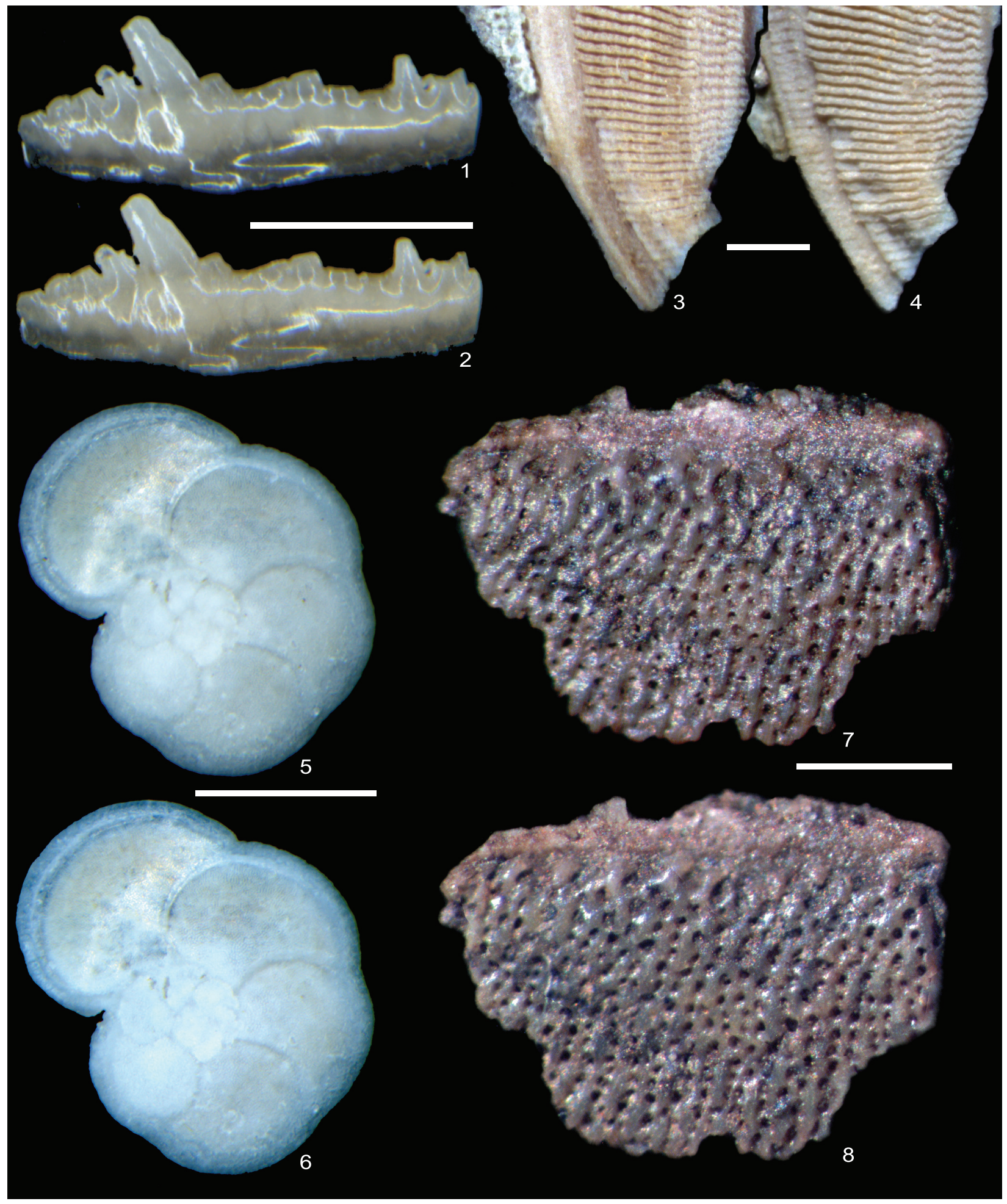

FIGURE 1. Optical images showing visual appearance of microfossils and micro-vertebrate remains before gold coating and after gold removal. 1, 2, Conodont element, Wurmiella excavata, Tramway Netherton, UK, Silurian: 1 before gold coating, 2 after removal. 3, 4, Sarcopterygian scale (probably Onychodus), Gogo Formation, Devonian, Australia: 3 before gold coating, 4 after removal. 5, 6, extant foraminiferan Globorotalia menardii (Caribbean Sea, $2900 \mathrm{~m}$ water depth): 5 before gold coating, 6 after removal. 7, 8, Fragment of dermal armour, probably heterostracan, Devonian, Welsh Borders, UK, 7 before gold coating, 8 after removal. Scale bar equals 500 $\mathrm{mm}$. 


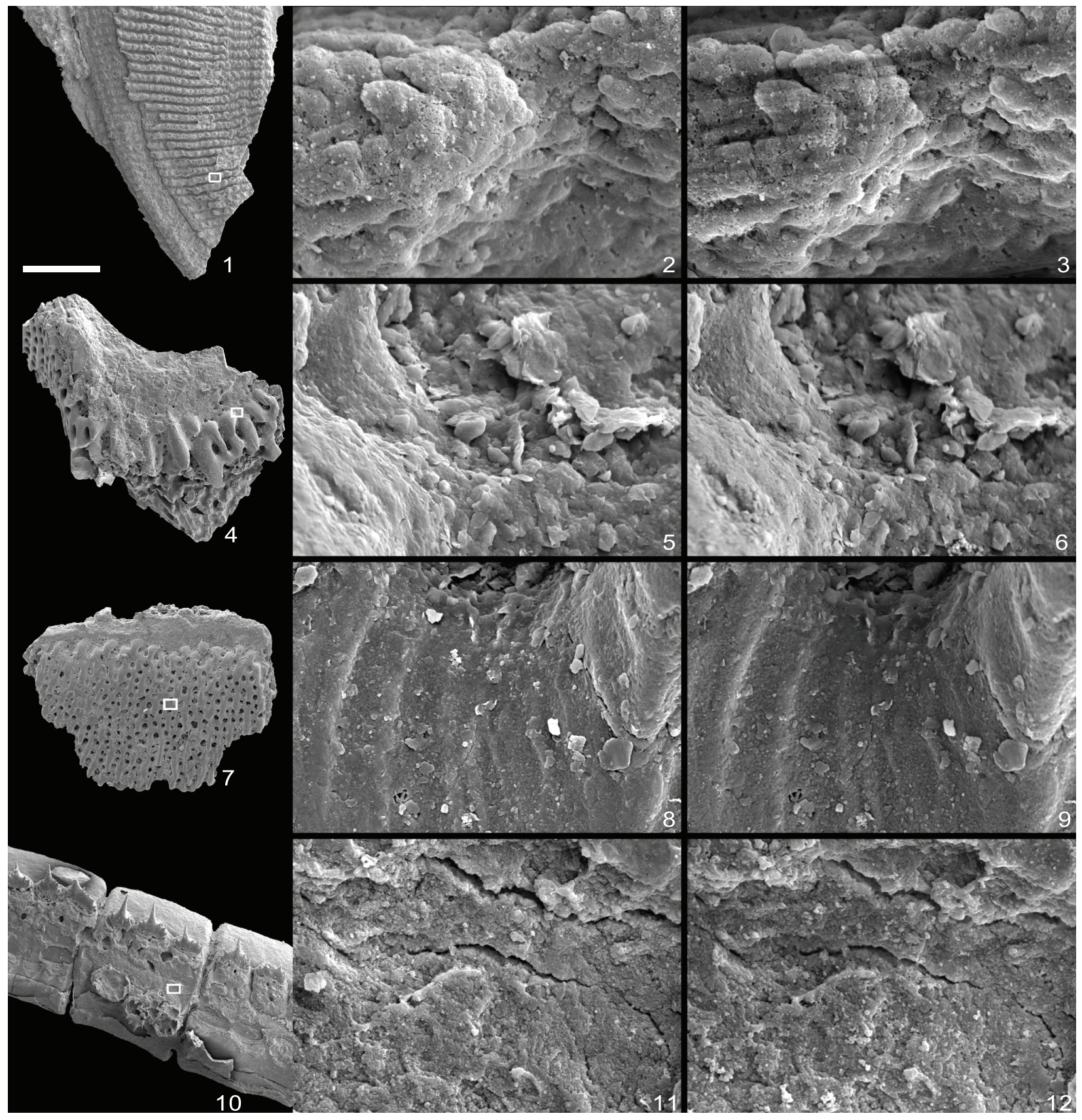

FIGURE 2. Scanning electron micrographs showing fine-scale surface textures of fossil microvertebrate remains before gold coating and after removal. 1-3, Sarcopterygian scale (probably Onychodus), Gogo Formation, Devonian, Australia: 2 before gold coating, 3 after removal (same specimen as Fig. 1.3,4). 4-6, Fragment of dermal armour, probably heterostracan (bony surface), Devonian, Welsh Borders, UK: 5 before gold coating, 6 after removal. 7-9, Fragment of dermal armour, probably heterostracan (dentine surface), Devonian, Welsh Borders, UK: 8 before gold coating, 9 after removal. 10-12, Sarcopterygian lepidotrichia (probably Onychodus), Gogo Formation, Devonian, Australia: 11 before gold coating, 12 after removal. White boxes in low-magnification images show location of high-magnification images. Low magnification scale bar equals $500 \mu \mathrm{m}$. Field of view of high-magnification images is $50 \mu \mathrm{m}$. 
to 12 hours, we found no evidence that solutions cause any chemical or physical damage to specimens. Longer durations of immersion in low concentrations of solution, up to 60 hours, also seem to have no effect, but it is advisable not to leave specimens in solution longer than is necessary. There was also no notable damage to the nonwater soluble adhesive. We expect this method, with appropriate choice of solvent type, to be equally effective on samples composed of other inorganic materials and with coatings of alternative metals, based on fundamental chemistry, detailed in Abbott et al. (2011a, 2011b). We suggest that anyone wishing to apply our method to alternative specimen compositions, coatings and adhesives should simply conduct a trial run first.

lodine concentrations of 0.05 and $0.1 \mathrm{~mol}$ $\mathrm{dm}^{-3}$ have been tested, making the process considerably faster. However, for concentrations of $0.1 \mathrm{~mol} \mathrm{dm}^{-3}$ surface etching was observed on one of the phosphatic specimens. In two instances the specimen had fallen off the SEM stub: one was attached with gum tragacanth, the other with a carbon tab. This error was probably due to handling, but may reflect loss of adhesive. Careful handling can probably avoid loss of nonwater soluble adhesive at least (see comments above regarding placement of specimens in liquid). Since lower concentrations were sufficient to dissolve gold coatings within 12 hours, we recommend not exceeding an iodine concentration of $0.02 \mathrm{~mol} \mathrm{dm}^{-3}$ (ca. $4.5 \mathrm{~g}$ of iodine per $\mathrm{kg}$ of Ethaline).

\section{ACKNOWLEDGEMENTS}

DJ and GF would like to thank N. Marsh for making us aware of each other. We thank $D$. Aldridge, D. Schmidt and P. Donoghue for providing specimens, S. Kearns for technical support and two reviewers, G. Miller and S. Leslie, for useful comments on an earlier version of the manuscript.
DJ is funded by Marie Curie International Outgoing Fellowship PIOF-GA-2009-235868. MAP and LD are funded by Natural Environment Research Council Grant NE/G018189/1 (to MAP). JH is funded by the Engineering and Physical Sciences Research Council and The MCP Group.

\section{REFERENCES}

Abbott, A.P., Frisch, G., Hartley, J., and Ryder, K.S. 2011a. Processing of metals and metal oxides using ionic liquids. Green Chemistry, 13:471-481.

Abbott, A.P., Frisch, G., Gurman, S.J., Hillman, A.R., Hartley, J., Holyoak, F., and Ryder, K.S. 2011b. Ionometallurgy: designer redox properties for metal processing. Chemical Communications, 47:1003110033.

Crissman, R.S. and McCann, P. 1979. A technique to remove gold palladium from SEM samples. Micron, 10:32-38.

Golden, J. 1989. Golden oldies: Curating SEM specimens. Collection Forum, 5:17-26.

Hansen, H.J. 1968. A technique for removing gold from plated calcareous microfossils. Micropalaeontology, 14:499-500.

Leslie, S.A. and Mitchell, J.C. 2007. Removing gold coating from SEM samples. Palaeontology, 50:14591461.

Miller, C.G., Cornish, L., Jones, C., Jones, C.G., and Henderson, A.S. 2004. A new laser method for cleaning micropalaeontological specimens. Journal of Micropalaeontology, 23:165-169.

Mills, A.A. 1988. Silver as a removable conductive coating for scanning electron microscopy. Scanning microscopy, 2:1265-1271.

Mills, A.A. 1989. A removable conductive coating for scanning electron microscopy. Studies in Conservation, 34:75-79.

Purnell, M.A. and Jones, D. in press. Quantitative analysis of conodont tooth wear and damage as a test of ecological and functional hypotheses. Paleobiology.

Purnell, M.A., Seehausen, O., and Galis, F. (2012). Quantitative three-dimensional microtextural analyses of tooth wear as a tool for dietary discrimination in fishes. Journal of the Royal Society Interface. doi: 10.1098/rsif.2012.0140. 


\section{APPENDIX}

The following recipe summarises the method for producing a standard quantity of the iodine solution:

To make ca. $1000 \mathrm{~cm}^{3}$ of Ethaline, mix $590 \mathrm{~g}$ of choline chloride and $530 \mathrm{~g}$ of ethylene glycol stirring at ca. $60^{\circ} \mathrm{C}$ until a clear homogenous liquid is formed.

Ethaline is stable and can be stored in glass or plastic containers for prolonged periods.

To make $100 \mathrm{~cm}^{3}$ of $0.02 \mathrm{~mol} \mathrm{dm}^{-3}$ iodine solution for gold removal, add $0.5 \mathrm{~g}$ of $\mathrm{I}_{2}$ to $100 \mathrm{~cm}^{3}$ of Ethaline, stirring at ca. $60^{\circ} \mathrm{C}$.

The iodine solution can be stored in a glass container for a week. 